\title{
Improving approximation by switching between two error functions
}

\author{
David Kaljun $^{1}$ and Janez Žerovnik ${ }^{1,2, *}$ \\ ${ }^{1}$ Faculty of Mechanical Engineering, University of Ljubljana \\ Aškerčeva 6, 1000 Ljubljana, Slovenia \\ 2 Institute of Mathematics, Physics and Mechanics \\ Jadranska 19, 1000 Ljubljana, Slovenia \\ E-mail:〈david@ekada.eu,janez.zerovnik@fs.uni-lj.si,janez.zerovnik@imfm.si〉
}

\begin{abstract}
The key to designing a LED luminaire is the choice of secondary optics. The design process can be improved using analytical models and optimization tools. A modified approximation error (evaluation function) is introduced in the standard analytical model for spatial light distribution. It is shown that the new evaluation function provides better quality and consistency compared to the standard evaluation function, and is a better practical approach to the optimization problem.
\end{abstract}

Keywords: optimization, LED luminaire, spatial light distribution, C-plane, RMS model, MAX model

Received: September 30, 2016; accepted: March 7, 2017; available online: March 31, 2017

DOI: $10.17535 /$ crorr.2017.0007

\section{Introduction}

A key part of any modeling process is whether a mathematical model describes a system accurately. Usually, approximation uses least squares and also the 2-norm is used to define the quality of approximation. In certain applications, however, other measures of approximation quality are more precise. This can be the case when designing a LED luminaire as the large difference between the desired and emitted light in one direction is observed easily, may be very annoying, and at the same time, and may end up as a poor design [12]. A luminaire that severely fails to fit the demanded light emission in only one direction may be completely useless, imagine for example the low beam of car headlamps. This failure, although likely to happen, may be overseen when checking the quality of approximation in 2-norm, and therefore it may be better to use the max-norm in such cases. However, analytical expressions for max-norm instead of the 2-norm are likely to be less convenient, as the corresponding functions may not be smooth. Therefore, there is a trade off between the seemingly more accurate model as opposed to faster approximation tools.

${ }^{*}$ Corresponding author. 
Note that the two models share the same zero-error approximations while the nearoptimal approximations may differ significantly. In this paper we investigate the possibility to switch among the models in order to enhance the convergence to near optimal approximations. The idea is not unlike the well-known variable neighborhood search heuristics [14], but it should be noted that here not only are the neighborhoods changed, but also the goal function. Preliminary results provided in this paper show that the method is promising as our results are comparable to those obtained using standard methods $[1,17]$. An important note is that the new approach was applied almost without or, more precisely, with substantially less tuning of the parameters compared to previous studies.

In the next section, we provide details of the application that has motivated this research, and Section 3 presents the model and evaluation functions. In Section 4, a brief description of the algorithms is provided. In Section 5, we describe the experiment and provide results, and discuss the conclusions in the last section.

\section{Motivation and previous work}

In recent years, LED (light emitting diode) technology in light sources has dramatically improved and consequently, its use has grown exponentially. The major reasons for success of LEDs is lower energy consumption, and endless possibilities of providing the light engine design (a combination of light source and optical elements). The latter enables the optics designer to build a lighting system that delivers light to an environment in a fully controlled fashion. However, numerous possible designs lead to new problems in choosing an optimal design depending on different goals such as optimization of energy consumption, production costs, and, last but not least, light pollution in an environment. The design process may be improved by using analytical models and optimization tools. This naturally opens up several research avenues. For example, when aiming to design a luminaire with a prescribed light emitting distribution, it may be cost-efficient to combine premanufactured LED lenses available on the market instead of producing special LEDs. This can be achieved using a software program that identifies combinations providing distributions close to what is desired [5]. It is important to have error free or at least very good approximations of the basic lenses, and methods that are stable, in the sense that they are not too sensitive to noise in the presentation of the input. Hence a model is needed containing the basic elements, i.e. a single LED luminaire with secondary optics. An adequate model provides design optimization and automatization - a highly ambitious goal that calls for further study (see [5] for a pilot experiment).

Here we limit our interest to a discussion on the basic model for LEDs with secondary optics. First, we recall the preliminary results. It is known that the spatial light distribution of some LED lenses can be approximated by a sum of a small number of certain basis functions [15]. The model was successfully applied to LEDs with secondary optics attached and symmetric light distribution [7] showing with sufficiently good approximations (RMS error below 5\%) obtained using a sum of only three functions $(K=3)$. The model was slightly modified in [7] where a new normalizing parameter was introduced, and consequently, all other parameters have known values in fixed intervals. It may be interesting to note that due to symmetries 
of the examples, $K=3$ is sufficient for both applications [7, 15]. In the general case, we expect that $K>3$ functions are necessary for sufficiently good approximations, and in optimizing a luminaire design, it becomes necessary to have an idea as to how large the parameter $K$ can increase in order to assure that the light distribution fits the desired (and/or standard) light distribution pattern sufficiently well.

In [7], several heuristic algorithms that describe the spatial light distribution of an LED with its attached secondary optical elements, $[6,8,9]$ were applied providing sufficiently good results. The tested algorithms include local search algorithms with different neighborhood definitions, as well as genetic and hybrid algorithms. Later, the results were improved by postprocessing using Newton's method [10]. Based on the remark of the reviewer from a previous report [8], we have considered the problem to be a nonlinear least squares problem in which variables can be separated [11], and have achieved a dramatic improvement in convergence speed that enables consideration of arbitrary distributions which are not necessarily symmetric. Originally, the model was applied to symmetric distributions which is equivalent to an approximation task on a single C-plane (See Fig. 1). Practical instances of the problem however typically involve examples without symmetry. The general method applied here approximates the entire spatial light distribution of one intersection half plane (C-plane) at a time. Thus, we approximated over 25 C-planes with 181 data points (polar angles) per C-plane, for every spatial light distribution. Altogether, we approximated over four thousand data points.

In all previous work $[6,8,9]$, the algorithms work with the same approximation error function which is the standard real mean square or RMS error. If we look at the definition of the RMS error, we see that it is designed in a way that hides high or low peak errors and provides an average estimate of the error. This is fine as long as we expect smooth input data with very little differences between data points. But in the field of visible light optics, we can also expect data with high differences. That is why we proposed an alternate approximation error function which we call the MAXe error [12] or, in other words, the infinite norm. The new approximation error function differs from the old one in that it does not give an average of the error over data points on some C-plane but rather the maximum error in that C-plane. In this way, we are careful not to ignore any initial data. As shown in [12], the new approximation error approach provides good results that are more reliable, and thus most probably is the better approach in the future, given that low MAXe error approximation implies the solution guarantees low deviation in any direction. On the other hand, as the algorithms used in evaluating the new model were not extensively tuned, in particular, much less time given to tuning compared to the original model, the best results achieved in the first model still seemingly outperform the later.

This motivates further study of the new model, in particular, the design and tuning of optimization algorithms. 


\section{Mathematical model and evaluation functions}

We search for an approximation of the Luminous intensity $I(\varphi ; \mathbf{a}, \mathbf{b}, \mathbf{c})$ at a polar angle of $\varphi$ in the form

$$
I(\varphi ; \mathbf{a}, \mathbf{b}, \mathbf{c})=I_{\max } \sum_{k=1}^{K} a_{k} \cos ^{c_{k}}\left(\varphi-b_{k}\right)
$$

where $K$ is the number of functions to sum and $a_{k}, b_{k}, c_{k}$ are the function coefficients that we search for. For brevity, coefficients are written as vectors $\mathbf{a}=$ $\left(a_{1}, a_{2}, \ldots, a_{K}\right), \mathbf{b}=\left(b_{1}, b_{2}, \ldots, b_{K}\right)$, and $\mathbf{c}=\left(c_{1}, c_{2}, \ldots, c_{K}\right)$. The interval ranges of the coefficients are: $\mathbf{a} \in[0,1]^{K}, \mathbf{b} \in[0,90]^{K}$ and $\mathbf{c} \in[0,100]^{K}$. When using discrete optimization algorithms, the parameter values are: $\mathbf{a} \in[0,0.001,0.002, \ldots, 1]^{K}$, $\mathbf{b} \in[-90,-89.9,-89.8, \ldots, 90]^{K}$, and $\mathbf{c} \in[0,1,2, \ldots, 100]^{K}$. Two restrictions on the model should be noted. First restriction emerges from the LEDs physical design. As the LED can not emit any light to the back side (the upper hemisphere), all values that are calculated at angle $\left(\varphi-b_{k}\right)$ greater than $180^{\circ}$ equal 0 . The second restriction is due to the slightly unusual description of the light distribution in standard files such as Elumdat (file extension .ldt) [16] and Iesna (.ies) [3]. These files present measured candela values per angle $\varphi$ on so called C-planes which can be observed on Figure 1. But a C-plane is actually only one half of the corresponding cross-section and does not describe the other half. However, from physical point of view the impact from the other half of the cross-section has to be considered.

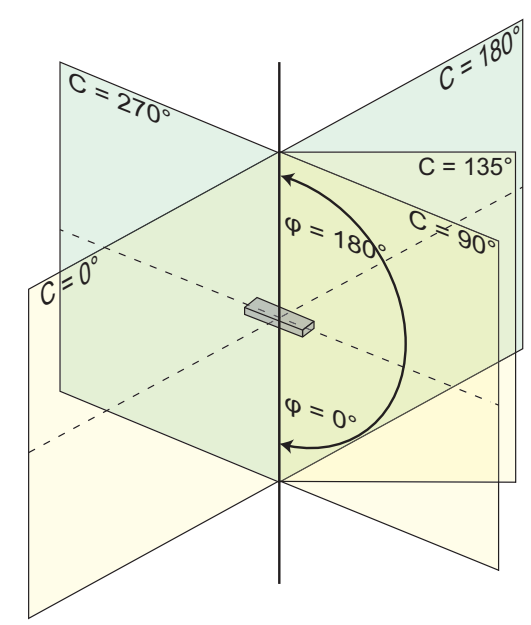

Figure 1: C-planes according to standard. C-planes angles : $0-360-\varphi$ angles : 0 to 180 degrees

As mentioned in the introduction, the impact of introducing a new evaluation function is investigated in this paper. That is why we define two functions that evaluate the goodness of fit differently. The first is the root mean square error (RMS), formally defined by the expression: 


$$
R M S(\mathbf{a}, \mathbf{b}, \mathbf{c})=\sqrt{\frac{1}{N} \sum_{i=1}^{N}\left[I_{m}\left(\varphi_{i}\right)-I\left(\varphi_{i}, \mathbf{a}, \mathbf{b}, \mathbf{c}\right)\right]^{2}}
$$

where $R M S$ represents the error of the approximation. Later in tables the relative RMS error (RMSp) defined by equation (4) is reported. Here, $N$ is the number of measured points in the input data, $I_{m}\left(\varphi_{i}\right)$ the measured Luminous intensity value at the polar angle $\varphi$ from the input data, and $I\left(\varphi_{i}, \mathbf{a}, \mathbf{b}, \mathbf{c}\right)$ the calculated luminous intensity value at the given polar angle $\varphi$.

The second alternative evaluation function used here is formally written as:

$$
\operatorname{MAXe}(\mathbf{a}, \mathbf{b}, \mathbf{c})=\max _{i}\left[I_{m}\left(\varphi_{i}\right)-I\left(\varphi_{i}, \mathbf{a}, \mathbf{b}, \mathbf{c}\right)\right]
$$

where $M A X e$ represents the maximum error, and all other variables are as defined in equation (2).

This gives two models which we refer on as Model 1 or RMS model and Model 2 or MAX model.

The results are provided in terms of relative $R M S$ and $M A X e$ error that are defined by equations (4) and (5). The reason for introducing (4) originaly was that the data in various datafiles available are not scaled, and the consequently the value $I_{\max }$, the maximal measurement, may differ significanlty among the lenses. Therefore a scaling factor $N /\left(\sum_{i=1}^{N} I_{m}\left(\varphi_{i}\right)\right)$ was introduced corresponding to average measurement. For easier comparison, a scaling factor is also applied to the $M A X e$ approximation error.

$$
\begin{gathered}
R M S_{p}(\mathbf{a}, \mathbf{b}, \mathbf{c})=\frac{100 * N * R M S(\mathbf{a}, \mathbf{b}, \mathbf{c})}{\sum_{i=1}^{N}\left[I_{m}\left(\varphi_{i}\right)\right]}[\%] \\
M A X e_{p}(\mathbf{a}, \mathbf{b}, \mathbf{c})=\frac{100 * M A X e(\mathbf{a}, \mathbf{b}, \mathbf{c})}{I_{\max }}[\%]
\end{gathered}
$$

Clearly, the optimal solutions (zero-error approximations) in both models coincide. However, the near optimal approximations may be very different. This is illustrated by the results in Table 1 where we compare solutions obtained from the two models. Here associated with each model is a version of an optimization algorithm where we only change the cost function that is to be minimized. The cost functions are, naturally, $R M S$ in the RMS model and $M A X e$ in the MAX model. (The optimization algorithm used is a local search type algorithm IF that is outlined later in more detail).

Looking at Table 1, the new model (MAX model) seems to be clearly inferior, but the main reason is of course that the solutions are evaluated differently. Namely, a fair comparison is to check the quality of solutions with the same error function. Here, the results of RMS model are clearly better when comparing the RMS error (first column in Table 2 compared to the second column in Table 1). In other words, optimization in Model 2 (applying the MAX cost local search) did not provide a better result compared to the original optimization method. On the other hand, the situation is not so clear when considering the MAX error (Table 2, second column). 
Here, in some cases MAX-based optimization is superior but on the other hand, RMS-based optimization in Model 1 in several cases provides better results also in terms of the MAX error. However, it is premature to draw any conclusions from the results provided here. More examples are given in [12] which shows interesting behavior that forbids any simple conclusions concerning the superiority of either model or algorithm.

Based on this observation, it may be a good idea to switch between the models and ask as to whether this may bring about any improvement in approximation quality or convergence speed. Recall that in introducing the second model, the error derived using the MAX norm is based on practical considerations [12]. However, the idea may be useful also in other applications where some other cost functions may be of interest.

In the next section, we will briefly outline the algorithms used in the experiments.

\begin{tabular}{|c|c|c|c|c|c|c|}
\hline & \multicolumn{3}{|c|}{$\begin{array}{c}\text { MAX model } \\
\text { MAX error }\end{array}$} & \multicolumn{3}{c|}{$\begin{array}{c}\text { RMS model } \\
\text { RMS error }\end{array}$} \\
\hline Lens & Best & Average & Worst & Best & Average & Worst \\
\hline C10818 & 5.0801 & 9.2589 & 14.2687 & 2.1021 & 3.3288 & 4.8276 \\
\hline C10949 & 3.1546 & 5.0670 & 7.1241 & 1.2023 & 2.1319 & 2.9317 \\
\hline CA11416 & 3.9740 & 6.0391 & 9.6082 & 1.5784 & 2.6409 & 4.5131 \\
\hline CA11426 & 5.6641 & 8.8768 & 15.089 & 1.9361 & 3.5252 & 6.4964 \\
\hline CA12050 & 3.5539 & 6.6315 & 11.1721 & 1.5788 & 2.8181 & 4.5389 \\
\hline CA12087 & 5.3171 & 13.2861 & 37.4918 & 2.1490 & 5.4651 & 14.1541 \\
\hline
\end{tabular}

Table 1: Comparison of the models

\begin{tabular}{|c|c|c|c|c|c|c|}
\hline & \multicolumn{3}{|c|}{$\begin{array}{c}\text { MAX model } \\
\text { RMS error }\end{array}$} & \multicolumn{3}{c|}{$\begin{array}{c}\text { RMS model } \\
\text { MAX error }\end{array}$} \\
\hline Lens & Best & Average & Worst & Best & Average & Worst \\
\hline C10818 & 2.6412 & 5.2852 & 8.6834 & 5.4280 & 8.7847 & 12.9902 \\
\hline C10949 & 1.9277 & 2.9424 & 4.5883 & 3.0165 & 5.6989 & 8.7168 \\
\hline CA11416 & 2.3757 & 3.353 & 5.2343 & 4.1378 & 7.3093 & 13.3517 \\
\hline CA11426 & 2.7196 & 4.8998 & 8.5563 & 5.5003 & 9.8205 & 22.8541 \\
\hline CA12050 & 1.9821 & 3.7464 & 6.7437 & 3.9828 & 7.6927 & 14.8069 \\
\hline CA12087 & 2.8540 & 7.6331 & 21.6723 & 6.0859 & 19.4859 & 64.3265 \\
\hline
\end{tabular}

Table 2: Models comparison - cont

\section{The algorithms}

In previous work $[5,6,7,8,9,10]$, the model described above was applied in conjunction with several custom built algorithms based on local search heuristics and certain meta-heuristics. The algorithms implemented in previous studies include a steepest descent algorithm, two iterative improvement algorithms with different neighbourhoods and two genetic algorithms, including a standard and a hybrid one in which the best individuals of every generation are optimized with the iterative improvement algorithm. 
The basic local search (iterative improvement with fixed neighborhood) is conceptually very simple and a well-known heuristics method $[1,17]$. Furthermore, it appears to be among the best algorithms among the applications we analysed in this paper [9], hence we decided to use this algorithm in the initial study, thus focusing on the effects of switching among two cost functions. The algorithm (standard IF) and its new variant (multi-evaluation IF) are explained briefly below.

Standard multi-start IF. The multi-start iterative improvement with fixed neighborhood (IF) first initializes several initial solutions. The initial solutions are randomly chosen from the whole search space. The initial solutions are then optimized using iterative improvement. At each step, the algorithm randomly chooses a neighbor, and immediately moves to the neighbor if its cost value is better than the current cost value. The neighbors of $\left(a_{1}, b_{1}, c_{1}, a_{2}, b_{2}, c_{2}, a_{3}, b_{3}, c_{3}\right)$ are $\left(a_{1} \pm d a, b_{1} \pm d b, c_{1} \pm d c, a_{2} \pm d a, b_{2} \pm d b, c_{2} \pm d c, a_{3} \pm d a, b_{3} \pm d b, c_{3} \pm d c\right)$, where $d a=0.01, d b=1$, and $d c=\frac{I_{\max }}{10}$. (Hence, there are 512 neighbors.) If no better neighbor is found after 1000 trials, it is assumed that no better neighbor exists. In this case the algorithm morphs the neighborhood by changing the step according to the formula $d_{i+1}=d_{i}+d_{0}$. More precisely, $d a_{i+1}=d a_{i}+d a_{0}$ where $d a_{0}$ is the initial step value. Analogously for $d b$ and $d c$. The algorithm stops when the number of generated solutions reaches $T_{\max }$.

Multi-evaluation multi-start IF has one parameter, $i$. The difference in the standard multi-start IF is that after $i$ changes of neighborhoods, the cost function is switched. If there is still no better solution, the algorithm changes the evaluation function from $R M S$ to $M A X e$, resetting the neighborhood, and the search is restarted in above described manner, the only difference being the evaluation function. If there is no better solution after $i$ changes of neighborhoods, the evaluation function is switched again, and so on. The algorithm stops when the number of generated solutions reaches $T_{\max }$.

\section{Experiments}

\subsection{Set-up and data-set}

As there are no standard benchmark data for the problem, we chose the same data set used in our previous experiments to make at least a comparison with our previous results. The choice of the dataset also shows that the algorithms are useful in real life scenarios.

We chose 9 different asymmetrical lenses to be used with a CREE XT-E series LED, from one of the worlds leading lens manufacturers, LEDIL from Finland. We acquired the photometric data from LEDIL's online catalog [13]. The data was provided in .ies format, which we then converted to a vector list and is more suitable for use in our algorithms. LEDIL measurements of the lenses gave a polar precision of $1^{\circ}$ on $25 \mathrm{C}$-planes. This means that from every .ies file, we extracted 2275 vectors. The experiment was set up so that all the lenses were approximated over all 25 C-planes. Each C-plane has the time limit $T_{\max }$ set to four million iterations. Each iteration is defined as the evaluation of the current set of parameters using the 
current evaluation function. In real time terms, this means that every lens was approximated in 30 minutes on a Core I7-4790K CPU @ $4.4 \mathrm{GHz}$. Given that the I7 $\mathrm{CPU}$ has four physical cores, the approximation in a multi-core setup of all twelve lenses takes a little more than two hours.

\subsection{Results - the first experiment}

The lenses were approximated over all 25 C-planes. In the tables (3, 4, and 5), the error on the best and the worst C-plane and the average result are obtained after four million iterations, comparing the standard IF with the multi-evaluation IF algorithm $(i=3)$. The best results after $4 \mathrm{M}$ iterations are written in bold.

\begin{tabular}{|c|c|c|c|c|c|c|}
\hline & \multicolumn{3}{|c|}{ Standard IF } & \multicolumn{3}{c|}{ Multi Evaluation IF } \\
\hline Lens/Iter & $\mathbf{1 0 0 K}$ & $\mathbf{1 M}$ & 4M & $\mathbf{1 0 0 K}$ & $\mathbf{1 M}$ & 4M \\
\hline CA13299 & 6.9718 & 4.5349 & 3.9896 & 3.7821 & 2.8903 & $\mathbf{2 . 8 9 0 3}$ \\
\hline CA13300 & 9.065 & 3.6753 & $\mathbf{3 . 3 0 8 9}$ & 4.7169 & 3.6351 & 3.6277 \\
\hline CA13805 & 5.7029 & 3.8884 & 3.7728 & 4.6041 & 3.2814 & $\mathbf{3 . 2 8 1 4}$ \\
\hline C10818 & 7.6407 & 3.826 & 3.6965 & 6.3525 & 2.8602 & $\mathbf{2 . 8 6 0 2}$ \\
\hline C10949 & 3.1882 & 1.8401 & $\mathbf{1 . 7 9 6}$ & 2.8224 & 2.0116 & 2.0116 \\
\hline CA11416 & 2.9323 & 2.1406 & 2.1387 & 2.2671 & 1.8862 & $\mathbf{1 . 8 8 6 2}$ \\
\hline CA11426 & 4.2799 & 3.5875 & 3.5053 & 6.1171 & 3.4659 & $\mathbf{3 . 4 6 5 9}$ \\
\hline CA12050 & 1.8933 & 1.7851 & $\mathbf{1 . 7 6 0 7}$ & 1.9922 & 1.7739 & 1.7739 \\
\hline CA12087 & 7.8819 & 2.3436 & $\mathbf{2 . 3 3 6 2}$ & 9.0052 & 3.0146 & 3.0146 \\
\hline Komb1 & 5.9555 & 4.0296 & $\mathbf{4 . 0 2 3 9}$ & 4.9804 & 4.2286 & 4.2286 \\
\hline Komb2 & 3.4397 & 3.3563 & 3.3563 & 3.3877 & 3.1698 & $\mathbf{3 . 1 6 9 8}$ \\
\hline
\end{tabular}

Table 3: Best RMSp results, Standard IF versus Multi Evaluation IF

\begin{tabular}{|c|c|c|c|c|c|c|}
\hline & \multicolumn{3}{|c|}{ Standard IF } & \multicolumn{3}{c|}{ Multi Evaluation IF } \\
\hline Lens/Iter & 100K & 1M & 4M & 100K & 1M & 4M \\
\hline CA13299 & 40.942 & 32.1781 & 32.1781 & 32.369 & 29.3404 & $\mathbf{2 2 . 7 2 5 8}$ \\
\hline CA13300 & 51.0319 & 44.0651 & 44.0651 & 47.3095 & 31.6158 & $\mathbf{3 1 . 6 1 5 8}$ \\
\hline CA13805 & 28.8676 & 28.3285 & 28.3285 & 28.152 & 13.1184 & $\mathbf{1 3 . 1 1 8 4}$ \\
\hline C10818 & 135.163 & 135.163 & 135.163 & 43.3599 & 39.6064 & $\mathbf{3 9 . 6 0 6 4}$ \\
\hline C10949 & 7.888 & 7.8858 & 7.8858 & 7.7721 & 4.5279 & $\mathbf{4 . 5 2 7 9}$ \\
\hline CA11416 & 12.365 & 7.5858 & 7.5858 & 10.9348 & 6.6492 & $\mathbf{6 . 6 4 9 2}$ \\
\hline CA11426 & 114.139 & 114.139 & 114.139 & 59.2504 & 46.3456 & $\mathbf{4 6 . 3 4 5 6}$ \\
\hline CA12050 & 32.2305 & 32.2274 & 32.2274 & 26.2531 & 12.1922 & $\mathbf{1 2 . 1 9 2 2}$ \\
\hline CA12087 & 116.168 & 116.168 & 116.168 & 96.217 & 46.0797 & $\mathbf{4 6 . 0 7 9 7}$ \\
\hline Komb1 & 50.5046 & 18.3178 & 18.3178 & 27.2 & 14.7034 & $\mathbf{1 4 . 7 0 3 4}$ \\
\hline Komb2 & 28.789 & 28.7216 & 28.7214 & 28.7406 & 16.4413 & $\mathbf{1 6 . 4 4 1 3}$ \\
\hline
\end{tabular}

Table 4: Worst RMSp results, Standard IF versus Multi Evaluation IF

In short, the experiment shows that switching among the cost functions provides better results. While the best results are in some cases obtained by the standard IF, the version that switches among the cost functions is clearly better when considering the worst and average solutions, thus we may say that it gives a more robust optimization method. 


\begin{tabular}{|c|c|c|c|c|c|c|}
\hline & \multicolumn{3}{|c|}{ Standard IF } & \multicolumn{3}{c|}{ Multi Evaluation IF } \\
\hline Lens/Iter & 100K & 1M & 4M & 100K & 1M & 4M \\
\hline CA13299 & 15.3567 & 10.7127 & 10.6134 & 13.1771 & 9.0846 & $\mathbf{8 . 3 0 4 1}$ \\
\hline CA13300 & 21.7651 & 11.1328 & $\mathbf{1 1 . 0 0 1 3}$ & 17.4506 & 12.1059 & 11.0836 \\
\hline CA13805 & 13.1719 & 9.4713 & 9.2052 & 9.7218 & 6.8671 & $\mathbf{6 . 8 6 6 7}$ \\
\hline C10818 & 26.9771 & 17.2709 & 17.1556 & 21.8802 & 11.9307 & $\mathbf{1 1 . 9 2 8 2}$ \\
\hline C10949 & 5.0333 & 3.3956 & 3.2434 & 4.2915 & 3.1271 & $\mathbf{3 . 1 2 7 1}$ \\
\hline CA11416 & 6.3793 & 3.9897 & 3.965 & 5.3212 & 3.4003 & $\mathbf{3 . 4 0 0 2}$ \\
\hline CA11426 & 36.5325 & 21.6352 & 21.1202 & 24.6908 & 17.091 & $\mathbf{1 7 . 0 9 1}$ \\
\hline CA12050 & 10.6401 & 7.4747 & 7.2966 & 8.6917 & 5.0087 & $\mathbf{5 . 0 0 8 3}$ \\
\hline CA12087 & 44.1952 & 25.2746 & $\mathbf{2 2 . 7 2 5 3}$ & 35.8289 & 24.882 & 24.8816 \\
\hline Komb1 & 16.127 & 8.6716 & 8.5033 & 11.9709 & 7.8635 & $\mathbf{7 . 8 6 3 5}$ \\
\hline Komb2 & 13.473 & 9.7719 & 9.6595 & 9.2142 & 7.3049 & $\mathbf{7 . 2 7 9 9}$ \\
\hline
\end{tabular}

Table 5: Average RMSp results, Standard IF versus Multi Evaluation IF

\subsection{Results - the second experiment}

The choice of the parameter value, $i=3$, was arbitrary and based on our intuition. In the second experiment, we briefly investigated the importance in choosing a value of parameter $i$. The multi-evaluation IF algorithm was run with various parameter values including $i=1,5,7,10$, and 15 . As before, $T_{\max }$ was set to 4 million iterations on each C-plane.

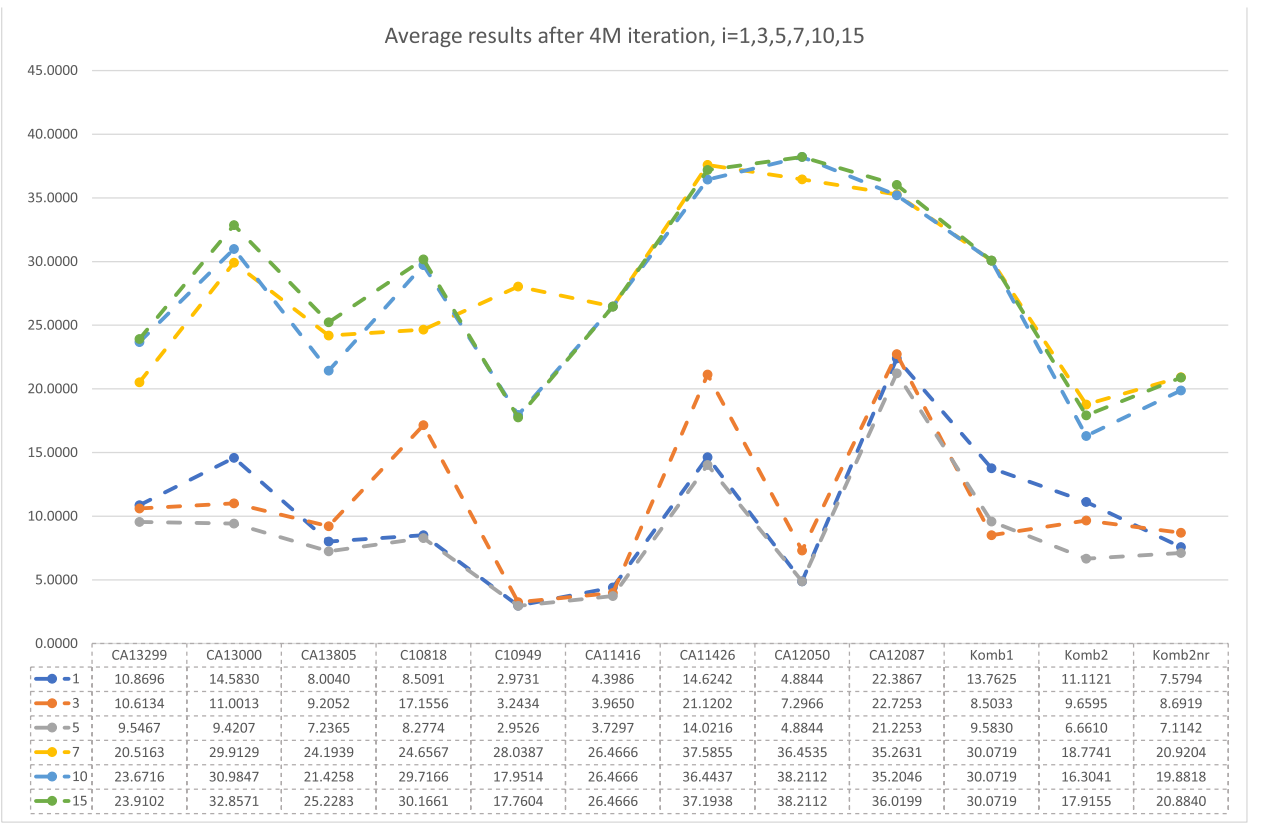

Figure 2: Average RMSp of Multi Evaluation IF, switching between RMS and MAX, variation of parameter $i$

Figure 2 shows that the smaller $i$ values are clearly superior to higher $i$ values. Results on the best and the worst C-planes are similar and are not appear as unexpected, hence we will not show them due to space limitations. As the number 
of experiments is limited, in conclusion we only say that it indicates that switching among the cost functions on this dataset proved to be useful and deserves further attention in future studies.

\subsection{Results - the third experiment}

In our application, that was the motivation behind the original experiment, we had two natural cost functions corresponding to definition of a good approximation. The first is the popular RMS error ( $L_{2}$ norm) and the second is the MAX error ( $L_{\infty}$ norm). However, the idea of switching among the cost functions may be useful in certain situations, where other error function(s) may provide a meaningful alternative to measure quality of approximation. For example, the sum of absolute errors $\left(L_{1}\right.$ norm) or any other $L_{p}$ norm, and perhaps some other measure, may be a reasonable choice. As suggested by the reviewer, we conducted a preliminary test for the $L_{1}$ case on our dataset. In the last experiment, the algorithms are the same as before, only the MAX cost function was replaced by the SUM cost function. The results are found in Figure 3. The solution for quality seems to behave similarly as before, depending on the values of $i$. Curiously, $i=1$ and $i=5$ provide nearly the same results, so that their graphs seemingly overlap. A comparison of the results in Figure 3 and Figure 2 shows that the combination of MAX and RMS for the dataset was superior to combining SUM and RMS.

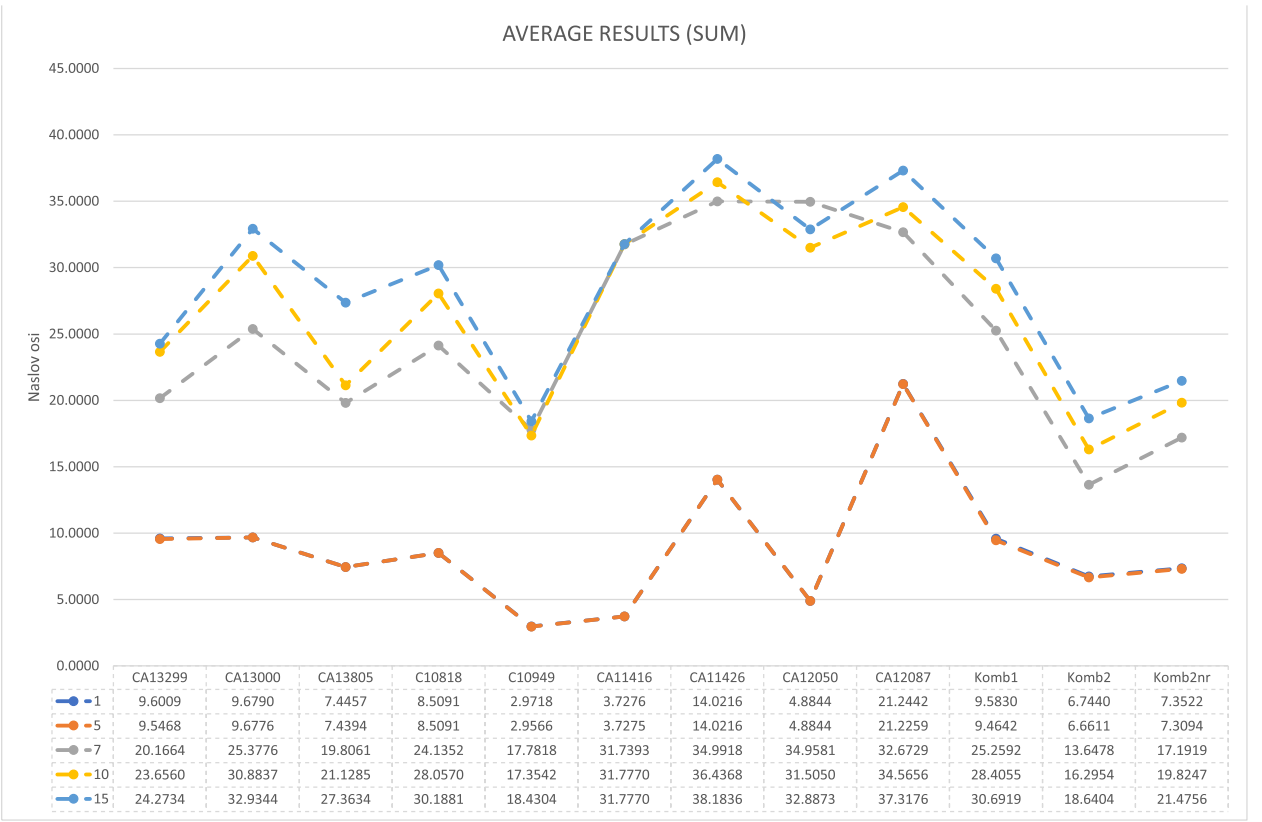

Figure 3: Average RMSp of Multi Evaluation IF, switching between RMS and SUM, variation of parameter $i$ 


\section{Discusion and conclusion}

First, we recall that the motivation for this study was to search for improved optimization tools in a particular application, for the purpose of designing a LED luminaire. As there are (at least) two natural choices for measuring the approximation quality, we compared the two corresponding models and also decided to test the idea of switching among the two models. The best results within the two models are obtained using algorithms incorporating the cost function of the chosen model, as expected. The advantage of the first model (RMS, using the $L_{2}$ norm norm) is the potential to improve the quality of solutions and speed of convergence compared with applying Newton's method. On the other hand, the second model (MAX, using $L_{\infty}$ norm) gives more reliable results and a guarantee that the solutions are of practical use. Combining both models, by switching the models during the optimization process, provides better results over a shorter time. We have concluded that the method for the realistic dataset in our application proved to be an improvement over the previously used methods.

The idea of switching among the cost functions regarded as a variation of the idea of variable neighborhood search, is much more general and deserves a more comprehensive approach. Here, we provided just a couple of preliminary experiments. We found that the value of paramater $i=3$ was a lucky choice, but, in general, this is definitely a parameter that should be tuned according to the problem and dataset in question. In the final experiment, we showed that using another norm $\left(L_{1}\right.$ instead of $L_{\infty}$ ) is relatively simple, but the combination did not give competitive results for our dataset. A number of possibilities remains unexplored (various cost functions, various parameter values) that may after some tuning lead to other useful techniques for local search based optimization.

The results should be viewed as preliminary given that applying more algorithms and having more time available for tuning the algorithm parameters would provide a fairer comparison, and should also be run on a larger dataset. Regardless of these limitations, we can conclude that switching between two evaluation functions provided an improvement when compared to the standard algorithm with a single evaluation function. Further experiments and development of algorithms are necessary, and open up a natural avenue of further research.

We also believe that the general idea of switching the error functions, or, among the cost functions, may be worth consideration in certain other applications. Of course, this has to be considered with much care, as it may lead to some confusion in which the formal definition of the optimization problem might be overlooked.

\section{Acknowledgement}

This work was supported in part by ARRS, the Research Agency of Slovenia, through grants P1-0285 and ARRS-1000-15-0510. The authors wish to sincerely thank the anonymous reviewers for constructive remarks that helped us considerably improve the presentation. 


\section{References}

[1] E. H. L. Aarts and J. K. Lenstra (1997). Local Search Algorithms. Chichester: John Wiley \& Sons.

[2] I. Ashdown (2001). Thinking photometrically part ii. In LIGHTFAIR 2001 PreConference Workshop.

[3] IESNA. Standard File Format for the Electronic Transfare of Photometric Data and Related Information LM-63-02. (ANSI, 2002).

[4] G.H. Golub and V. Pereyra (1973). The Differentiation of Pseudo-Inverses and Nonlinear Least Square Problems Whose Variables Separate. SIAM J. Numer. Anal. 16. 413-432.

[5] D. Kaljun (2016) Optical system design optimization of a LED luminaire. (Optimizacija načrtovanja optičnega sistema led svetilke). PhD thesis, University of Ljubljana (in Slovene).

[6] D. Kaljun and J. Žerovnik (2014). Local Search Optimization of a Spatial Light Distribution Model, In Šilc J. and Zamuda A. (eds.), Bioinspired Optimization Methods and their Application, Jožef Stefan Institute, Ljubljana, 81-91.

[7] D. Kaljun and J. Žerovnik (2014). Function fitting the symmetric radiation pattern of a LED with attached secondary optic. Optics Express 22, 29587-29593.

[8] D. Kaljun and J. Žerovnik (2014). On local search based heuristics for optimization problems. Croat. Oper. Res. Rev. 5, 317-327.

[9] D. Kaljun and D. Rupnik Poklukar and J. Žerovnik (2015). Heuristics for Optimization of LED Spatial Light Distribution Model. Informatica 39, 317-327.

[10] D. Kaljun and J. Petrišič and J. Žerovnik (2016). Using Newton's method to model a spatial light distribution of a LED with attached secondary optics. Strojniški vestnik - Journal of Mechanical Engineering 62, 307-317.

[11] D. Kaljun and T. Novak and J. Žerovnik. Improved Approximation of Spatial Light Distribution. PLOS ONE, accepted for publication.

[12] D. Kaljun and J. Žerovnik, An alternative model of the spatial light intensity distribution. Optics Express, preprint.

[13] Ledil Oy., http://www.ledil.com/products/?y, accessed on 14.09.2016.

[14] N. Mladenović and P. Hansen (1997). Variable neighborhood search, Comput. Operations Res. 24, 1097-1100.

[15] I. Moreno and C.-C. Sun (2008). Modeling the radiation pattern of leds. Optics express 16, 1808-1819.

[16] SIST, Light and lighting - Measurement and presentation of photometric data of lamps and luminaries - Part 1: Measurement and file format EN 13032-1:2004+A1:2012 (SIST, 2012).

[17] E.-G. Talbi (2009). Metaheuristics: From Design to Implementation. John Wiley \& Sons. 